\title{
Prótese Parcial Removível Flexível - revisão de literatura
}

\author{
Flexible Removable Partial Prótese - a literature review \\ Dentadura Parcial Removible Flexible - revisión de la literatura \\ Bruna Maria Gonçalves do PATROCÍNIO ${ }^{1}$ \\ Aline Moreira ANTENOR ${ }^{1}$ \\ Marcela Filié HADDAD ${ }^{2}$
}

${ }^{1}$ Aluna do Curso de graduação em Odontologia, Departamento de Odontologia Restauradora,

Faculdade de Odontologia, Universidade Federal de Alfenas (UNIFAL-MG), 37.130-000 Alfenas - MG, Brasil

${ }^{2}$ Cirurgiã-Dentista, Mestre e Doutora em Prótese,

Professora da Disciplina de Prótese Parcial Removível do Departamento de Odontologia Restauradora,

Faculdade de Odontologia, Universidade Federal de Alfenas (UNIFAL-MG), 37.130-000 Alfenas - MG, Brasil

\begin{abstract}
Resumo
Objetivo: Comparar as Próteses Parcias Flexíveis (PPFlex) com as convencionais (PPC), apresentando as vantagens, desvantagens, indicações e contraindicações esclarecendo os dentistas para que façam a indicação consciente e considerando as limitações deste tipo de prótese. Materiais e Métodos: Os termos "Próteses Parciais Removívies", "Próteses Flexíveis", "Resinas Flexíveis", "Edêntulos Parciais" e "Materiais para Próteses Parciais Removíveis" foram consultados nas bases de dados Pubmed, Bireme e Scielo. Assim, 35 arquivos foram selecionados para compor esta revisão. Resultados: As PPFlex representam uma alternative às PPCs para pacientes com grande exigência estética e alergia à liga de cromo-cobalto ou ao polimetilmetacrilato. As PPFlex são produzidas com um tipo especial de resina flexível e não apresentam grampos metálicos. Este material prove maior conforto e estética quando comparado às próteses convencionais que são feitas de resina acrílica e estrutura metálica. Conclusão: Embora haja uma popularidade progressiva deste material nos últimos anos, o fato de não conter um suporte oclusal e estrutura metálica cria incertezas quanto ao seu desempenho clínico, especialmente em arcadas de extremidade livre, onde a estabilidade depende da combinação de suporte entre dente e tecido mole.
\end{abstract}

Descritores: Prótese Parcial Removível; Prótese Parcial Temporária; Bases de Dentadura.

\begin{abstract}
Objective: To compare the flexible partial dentures (FPD) to conventional partial dentures (CPD), highlighting the advantages, disadvantages, indications and contraindications in the hope that more dentists may consider the conscious use and the limitations of this new kind of prothesis. Matherial and Methods: The terms "Removable Partial Dentures", "Flexible Prostheses", "Flexible Resins", "Partially Edentulous" and "Materials for Partial Removable Prosthesis" were searched in Pubmed, Bireme and Scielo databases. So, 35 files were selected to compose this review. Results: FPDs are alternative to CPDs for patients with high aesthetic requirements and allergic to alloys of chrome cobalt or polymethylmethacrylate. FPD is produced from a special type of flexible resin and it has no metal clasps. This material provides more comfort and aesthetics when compared to conventional prostheses that are made of acrylic resin and metal frame. Conclusion: Although there is a progressive popularity of this material in recent years, the fact that it does not contain an occlusal support and metal structure changes the enthusiasm to uncertainties regarding its clinical performance, especially in free-end arches, where stability depends on a combination of teeth and soft tissue.

Descriptors: Denture, Partial, Removable; Denture, Partial, Temporary; Denture Bases.
\end{abstract}

\section{Resumen}

Objetivo: Comparar las Dentaduras Parcias Flexíbles (PPFlex) con las Convencionais (PPC), presentar las ventajas, desventajas, indicaciones y contraindicaciones que aclaran los dentistas para hacer una declaración consciente y teniendo en cuenta las limitaciones de este tipo de prótesis. Materiales e Métodos: Los términos "Dentaduras parciales Removibles", "Dentaduras flexibles", "Resinas flexibles", "desdentados parciales" y "Materiales para prótesis parciales removibles" fueron encontrados en Pubmed, Bireme y Scielo Por lo tanto, se seleccionaron 35 archivos para componer esta revisión. Resultados: la PPFlex representan una alternativa a las PPC para los pacientes con altas exigencias estéticas y alergia a la aleación de cobalto-cromo o polimetilmetacrilato la PPFlex se producen con un tipo especial de resina flexible y no tiene cierres metálicos, el material proporciona una mayor comodidad y la estética en comparación con las prótesis convencionales que están hechos de acrílico y resina de estructura de metal. Conclusión: Aunque hay una popularidad progresivo de este material en los últimos años, el hecho de no contener un soporte oclusal y un marco de metal crea incertidumbre sobre su rendimiento clínico especialmente en los arcos de extremo libre, donde la estabilidad depende del apoyo combinación entre el diente y el tejido blando.

Descriptores: Dentadura Parcial Removible; Dentadura Parcial Provisória; Bases para Dentadura.

\section{INTRODUÇÃO}

Espera-se que no ano de 2030 ocorra um significativo aumento de expectativa de vida no mundo inteiro. Estima-se que o Brasil atingirá o número de 30 milhões de idosos em 2050. Assim a tendência é que a saúde bucal seja considerada cada vez mais importante como componente da saúde geral, visto que os indivíduos viverão mais tempo e uma fração expressiva da população desdentada é idosa ${ }^{1}$.

A reabilitação da função mastigatória e da aparência são objetivos importantes, principalmente quando os pacientes apresentam perda dentária extensa e possuem apenas alguns remanescentes dentais ${ }^{2,3}$. Assim, a busca de tratamentos por parte dos pacientes parcialmente desdentados está aumentando progressivamente. Dentre as opções de reabilitação e reposição dos dentes em arcadas parcialmente desdentadas pode-se citar a prótese dentária implantossuportada, a prótese parcial fixa e a prótese parcial removível (PPR). Alguns pacientes podem optar pela prótese inteiramente suportada por implantes; entretanto não são todos capazes de adotar os cuidados necessários para este tipo de reabilitação. Adicionalmente, o uso de implantes ou prótese fixa convencional tem um custo elevado demais para ser uma opção viável para todos os pacientes. Ainda que o uso de implantes dentários tenha aumentado como opção de tratamento, as próteses parciais removíveis (PPRs) 
permanecem sendo a primeira escolha para muitos pacientes e dentistas, evitando a necessidade de submeter o paciente a tratamentos cirúrgicos e protéticos longos, complexos e principalmente onerosos ${ }^{2,4,5,6}$.

As PPRs convencionais são utilizadas há décadas para a reabilitação de indivíduos parcialmente desdentados. Estas usam sistema de grampos fundidos em metal e promovem a reabilitação oral de forma satisfatória, porém não são estéticas, e por essa razão são menos desejadas como meio reabilitador por parte dos pacientes ${ }^{4,7}$.

Diante desta problemática surge uma nova possibilidade de reabilitação que tem ganhado visibilidade entre pacientes e dentistas nos últimos anos: as próteses flexíveis, que são constituídas de material maleável e de coloração semelhante à mucosa, sendo, portanto, estéticas e confortáveis. Elas também podem representar uma opção de tratamento para a reabilitação de indivíduos alérgicos ao polimetilmetacrilato ou ao cromo-cobalto, em casos de fraturas constantes de próteses convencionais, em pacientes com destreza manual reduzida e ainda em condições de microstomia, tórus palatino, fissura palatina ou rebordo em lâmina de faca ${ }^{4}$.

Embora esse tipo de prótese seja amplamente utilizado em alguns países e da crescente popularidade na mídia, não existem evidências clinicas ou estudos sobre os efeitos em longo prazo e eficácia das próteses flexíveis. Assim se essa prótese for indicada ou confeccionada de forma arbitrária, ou ainda se for usada de maneira inapropriada, o tratamento será insatisfatório, acarretando inúmeras consequências negativas para o paciente. Dessa maneira, o presente trabalho tem como objetivo compreender as diferenças entre próteses parciais removíveis convencionais e flexíveis, visando esclarecer o clínico geral a respeito da sua constituição, vantagens e desvantagens; indicações e contraindicações ou limitações de uso.

\section{MATERIAL E MÉTODO}

Foram pesquisadas nas bases de dados Pubmed, Bireme, Google acadêmico e Scielo os termos "Próteses Parciais Removíveis", "Próteses Flexíveis", "Resinas Flexíveis"; "Desdentados Parciais" e "Materiais para Prótese Parcial Removível". Foram encontrados 80 artigos e 28 livros sobre o assunto. Foram incluídos neste trabalho, artigos em língua portuguesa, inglesa e espanhola que abordassem a reabilitação de pacientes parcialmente desdentados por meio de próteses parciais removíveis convencionais e flexíveis, trabalhos de pesquisa e revisões de literatura sobre o mesmo tema. Foram excluídos trabalhos em idiomas diferentes dos citados acima e que não envolvessem relatos a respeito de reabilitação de arcadas parcialmente desdentadas. Desta maneira 35 arquivos foram selecionados para compor esta revisão.

\section{REVISÃO DA LITERATURA}

\section{○ MATERIAIS}

Os materiais utilizados atualmente para a construção de PPRs convencionais incluem ligas com bases de cobaltocromo, resina acrílica termoativada e dentes artificiais também acrílicos. A PPR convencional usa sistemas de grampos, que requerem, além de um planejamento ótimo e uma biomecânica eficiente, a utilização de materiais que possam conferir a prótese retenção e estabilidade sem prejudicar os tecidos de suporte ${ }^{8}$.
A liga a base de cobalto-cromo é a mais utilizada por ser resistente à corrosão e ao manchamento, apresentar baixa densidade, possuir compatibilidade com os tecidos bucais e apresentar custo relativamente baixo. Dentre as desvantagens deste material, pode-se listar dificuldade na definição de detalhes, de acabamento, de polimento e contração durante a fundição 9 .

Além dessas desvantagens, não menos importante é o fator estético, pois o fato de que o metal aparece em regiões visíveis faz com que as próteses convencionais sejam indesejadas pelos pacientes ${ }^{7}$.

As resinas acrílicas termoativadas são utilizadas industrialmente para a produção dos dentes artificiais e no laboratório de prótese para confecção das bases de PPRs convencionais. Representam o material de primeira escolha para confecção de bases porque apresentam estabilidade de cor e de propriedades óticas em condições normais, propriedades físico-químicas aceitáveis e facilidade de manipulação. Assim, vários materiais para a confecção de próteses já foram testados, como porcelana, alumínio e ainda assim, a resina acrílica é o material que reúne as propriedades físico-químicas e biológicas mais adequadas ${ }^{10}$.

Entretanto, algumas desvantagens podem ser apontadas como, por exemplo, a resina acrílica pode apresentar alterações e causar movimentação dos dentes artificiais durante o processo de polimerização; ou ainda, em alguns casos, pode apresentar porosidades ${ }^{6,11}$.

$\mathrm{O}$ aumento da preocupação estética e a busca pelo conforto levaram os pacientes a desejarem que as próteses parciais removíveis revelem o mínimo possível de estruturas metálicas e que sua textura seja agradável na boca. Assim, um novo conceito de próteses mais estéticas e agradáveis tem ganhado espaço na odontologia: as próteses flexíveis ${ }^{4}$.

As próteses flexíveis foram desenvolvidas em 1956, nos Estados Unidos, pela companhia Valplast ${ }^{\circledR}$, com o intuito de serem utilizadas por pacientes com alergia ao monómero de resina acrílica e para a construção de próteses flexíveis provisórias ${ }^{12,13,14}$. Elas têm ganhado popularidade entre dentistas e pacientes por serem confeccionadas sem metal, sendo, portanto, melhores esteticamente e mais confortáveis. Além disso, ganharam fama por substituírem as próteses convencionais em casos de alergia ao polimetilmetacrilato ou ao cromo-cobalto. Essas próteses não convencionais são constituídas de dentes usados em PPR, associados a uma resina termoplástica que constitui a base dessas próteses $\mathrm{s}^{4,7,10}$.

Um material termoplástico é um plástico que se torna maleável em determinada temperatura. As resinas flexíveis são vendidas na forma de grãos, disponíveis em tons diferentes, a saber: RC: Rosa Claro, RM: Rosa Médio, RE: Rosa Escuro, RR: Rosa Roxo, RV: Rosa Vermelho. A cor rosa médio é considerada a cor padrão ${ }^{15}$.

Existem variados tipos de resinas termoplásticas disponíveis como poliamidas, poliéster e policarbonatos; contudo, as poliamidas são as mais utilizadas devido a sua flexibilidade inerente ${ }^{16}$.

\section{POLIAMIDA}

As poliamidas, conhecidas genericamente por nylon, apresentam características vantajosas como alto padrão estético, a reprodução da cor da gengiva natural e também a superioridade no conforto relatado pelos pacientes. Devido ao fato de possuir alto nível de flexibilidade, a poliamida torna as próteses mais leves e finas ${ }^{4}$.

Quimicamente, os nylons são copolímeros de condensação formados por reação de uma diamina e um 
ácido dicarboxílico. Fazem parte da constituição: copolímeros de propileno, pigmentos e plastificantes. É importante salientar que a composição pode modificar de acordo com a marca o que pode trazer diferenças nas propriedades desses materiais. Por exemplo, diferenças na porcentagem de nylon incorporadas pelos fabricantes podem alterar as propriedades mecânicas referentes à resistência e à deformação ${ }^{4,16,17}$.

Algumas marcas, como a Valplast, têm 99,9\% do seu conteúdo de nylon 12. Essa constituição química faz com que as próteses feitas desse material sejam suaves, facilmente deformáveis e elásticas. Outras marcas, como Lucitone, são feitas de uma poliamida microcristalina, que tem a vantagem de ser resistente à quebra. Isso confere suavidade em comparação com as resinas de poliéster e de policarbonato. Em outras palavras, as poliamidas fornecem um ajuste bom e são menos propensas a se quebrar ${ }^{7}$.

No entanto, como qualquer material, as resinas de poliamida apresentam algumas deficiências como uma baixa resistência à tração e à flexão. Segundo Da $\mathrm{Costa}^{4}$, a resina Valplast sofre uma diminuição do seu módulo elástico e da sua resistência à flexão com o tempo mostrando que as variações as quais a resina é exposta dentro da boca tem influência direta nas características do material.

Outro ponto negativo é a instabilidade de cor. Testes laboratoriais relatam que a resina flexível sofre algumas alterações de cor ao longo do tempo. Com o envelhecimento ocorrem alterações cromáticas, adsorção de água e pigmentos, e oxidação. Assim, em longo prazo esses materiais podem perder parte de sua estética também ${ }^{4,18}$.

Um material interessante utilizado para construir próteses flexíveis de nylon é derivado do óleo de mamona. Esse biopolímero $100 \%$ natural é um material composto por uma cadeia de polímero estável que não contêm monômeros, e consequentemente não liberam componentes após polimerização. Portanto, reações alérgicas, como estomatite protética, são diminuídas em grande parte. Esta prótese de Nylon dá um aspeto mais natural quando colocada em boca $^{18}$.

Embora a poliamida seja um plástico aprimorado, esse material não é uma solução para a deficiência funcional dessas próteses, pois com o passar do tempo, os grampos de plástico flexível não resistem à mecânica da colocação e remoção diária da prótese e à pressão das forças mastigatórias que podem causar afastamento gengival e consequentemente reabsorção óssea exagerada. Adicionalmente a essas desvantagens também existem limitações associadas ao aumento da rugosidade da superfície e dificuldades de reembasamento e consertos devido à incapacidade de retenção química dos dentes de acrílicos a base da prótese $e^{4,10}$.

\section{POLIÉSTER}

Uma característica importante dos poliésteres é que eles têm boa adesão às resinas quimicamente ativadas. Isso significa que existe uma facilidade de reparo como, por exemplo, na adição de dentes perdidos. Algumas marcas de poliéster tem um polímero de polietileno conhecido como o principal material usado para fazer garrafas de plástico ${ }^{18}$.

Alguns pontos positivos incluem a boa aparência estética, funcionalidade e maior aderência do que as resinas de poliamida. Os pontos negativos incluem menor resiliência, mais susceptibilidade à abrasão e menor dureza em comparação com a resina de poliamida. Isso significa que ela é mais vulnerável, pois tem baixa resistência à fratura $^{11,19}$

\section{POLICARBONATO}

Os policarbonatos são produzidos a partir de melhorias nas resinas termoplásticas. Originalmente, ele foi produzido na área da odontologia para uso em PPRs convencionais. Alguns autores defendem que sua resistência à flexão é superior a poliamida e o poliésteres. É também menos suscetível a descoloração e na sua superfície é altamente resistente à abrasão. Ele apresenta menor absorção do que as resinas acrílicas, assim do ponto de vista da higiene o policarbonato é mais adequado ${ }^{18}$.

Esse material não provoca irritação ou reação alérgica. Uma característica inconveniente é o fato de que ele pode provocar descamação na mucosa e seu uso deve ser evitado como prótese imediata ${ }^{11}$.

\section{○ BIOMECÂNICA}

Para que uma PPR exerça bom funcionamento, deve respeitar três princípios biomecânicos: retenção, suporte e estabilidade, e para isso, as PPRs devem apresentar alguns elementos constituintes ${ }^{17,20,21}$.

\section{CONSTITUINTES DE UMA PRÓTESE PARCIAL REMOVÍVEL}

As PPRs convencionais apresentam elementos constituintes diferentes das PPRs flexíveis. Podem ser citados constituintes das PPRs Convencionais:

a) apoio oclusal: suporta e estabiliza a prótese no sentido ocluso-gengival transferindo a força mastigatória, que incide sobre os dentes artificiais, aos dentes pilares e permite que as demais partes da prótese ocupem a posição correta durante a função, evitando prejuízo às estruturas de suporte ${ }^{22}$.

b) grampos: são os elementos mecânicos do retentor extracoronário. São constituídos, basicamente, por retentor e opositor. O retentor tem sua ponta ativa flexível e esta é a única parte que deve se localizar completamente abaixo do equador protético com a função de garantir a retenção do aparelho durante a função. $\mathrm{O}$ opositor é completamente rígido e deve se posicionar acima do equador protético, para estabilizar e evitar movimentação ortodôntica sobre o dente pilar $^{22}$.

c) sela: estrutura constituída pela malha metálica, base de resina e dentes artificiais. Deve existir relação de íntimo contato entre a sela e o tecido fibromucoso, em especial em áreas de extremidades livres, para evitar injurias teciduais e garantir uma distribuição de forças adequada durante a função ${ }^{23}$.

d) conector maior: liga os elementos de um lado do arco dental ao outro, no qual se unem todas as partes das próteses direta ou indiretamente. Como características principais deve apresentar rigidez, para distribuir as cargas, resistir à tração e à fratura, não deve perturbar a fonética nem traumatizar a mucosa durante a instalação e remoção ${ }^{9}$.

e) conector menor: une o retentor à sela e/ou ao conector maior. Têm como função transmitir aos dentes de suporte as forças mastigatórias e contribuir para a rigidez do aparelho protético.

As PPRs flexíveis, por sua vez, apresentam os seguintes componentes ${ }^{16}$ :

a) base flexível corresponde à parte de PPR à qual os dentes artificiais estão ligados e que se apoia sobre a mucosa. Servem de suporte aos dentes, restauram a estética e função mastigatória;

b) grampos que também são feitos de material termoplástico flexível.

Então, pode-se dizer que no caso das PPRs Flexíveis, o suporte se dá através do contato da prótese com a superfície do dente e sua mucosa subjacente e somente a 
relação prótese-dente-mucosa é utilizada para evitar qualquer tipo de movimentação, oferecendo assim segurança para o paciente falar e mastigar de modo confortável. Assim, não são utilizados ganchos metálicos e não existem apoios, fato que elimina a necessidade de preparos dentais, porém, implica na ausência de transmissão de forças para os dentes pilares no sentido do seu longo eixo, podendo lesar o periodonto de sustentação ${ }^{6}$.

A distribuição de tensões nas próteses removíveis convencionais é controlada por elementos estruturais do desenho, especificamente com os apoios oclusais, conetores menores e planos guia. Esta ação na Prótese Flexível é realizada pela flexibilidade no conector principal comportando-se como um rompe-forças. A grande questão deste tipo de estrutura é a sua flexibilidade, que contraria totalmente os conceitos das PPRs convencionais. Em longo prazo, a flexibilidade das próteses parece atuar como um condicionador de tecidos, uma vez que o sutil movimento ao longo dos tecidos estimula a circulação sanguínea local ${ }^{24}$.

É desejável que os materiais constituintes da prótese apresentem uma resistência à flexão suficientemente alta para que não ocorra deformação permanente com as cargas aplicadas durante a mastigação ou com a inserção e remoção da prótese. Os materiais de base de prótese apresentam uma considerável deformação plástica antes de fraturar, mas a deformação além do limite proporcional resulta numa alteração permanente das suas dimensões, podendo interferir negativamente na retenção do aparelho, além de provocar lesões na mucosa subjacente. Valores menores de resistência à flexão das poliamidas resultam numa maior deformação permanente durante a mastigação, existindo o risco de reabsorção óssea devido à acumulação de stress vertical. Assim, recomenda-se que a base da prótese de poliamida seja reforçada com um esqueleto metálico para diminuir o risco de reabsorção óssea ${ }^{25}$.

Um estudo avaliou o impacto que as diferentes propriedades mecânicas poderiam ter na eficácia mastigatória, relatando que as próteses flexíveis possuíam menor eficácia mastigatória nos alimentos duros, não havendo diferenças para alimentos moles ${ }^{3}$.

Próteses produzidas a partir da técnica de injeção, como as resinas de poliamida, resultam numa menor contração de polimerização, produzindo assim uma prótese mais ajustada ${ }^{26}$.

Os materiais que constituem a prótese ao serem imersos num meio aquoso ficam susceptíveis à absorção de água, em que plastificantes e outros componentes solúveis integrantes da prótese podem escoar ao longo do tempo, enquanto a água e saliva são absorvidas à perda do plastificante juntamente com a água absorvida resulta em efeitos prejudiciais nas propriedades físicas e mecânicas, como alterações de cor, amolecimento do material, diminuição da rigidez, da resistência à flexão, da resistência à fadiga e da estabilidade dimensional, afetando assim a sua retenção, estabilização e longevidade ${ }^{27}$.

\section{○ INDICAÇÕES E CONTRAINDICAÇÕES}

Shaghaghien et al. $^{28}$ apresentaram as seguintes indicações para a utilização das PPRs Convencionais:

a) repor alguns dentes em um quadrante, ou nos dois quadrantes da mesma arcada;

b) servir como prótese provisória para repor dentes perdidos em uma criança (se necessário um novo aparelho para acompanhar o crescimento da criança);

c) repor dentes que faltam a pacientes que não toleram tratamentos longos como os de prótese fixa ou implantes; d) servir de tala para dar suporte a dentes periodonticamente envolvidos;

e) questões financeiras (as próteses removíveis são, por norma, mais acessíveis que os tratamentos fixos);

f) em casos de excessiva perda óssea, onde a colocação da prótese fixa ou implantes pode levar a comprometimentos estéticos não aceitáveis;

g) como provisórias, em pacientes com extrações recentes e pacientes em tratamentos que requerem osseointegração.

São contraindicações ou limitações de uso das PPRs Convencionais ${ }^{29}$ :

a) pacientes que não apresentam um controle adequado da higiene bucal;

b) indivíduos com comprometimento mental ou motor.

São Indicações para PPR Flexível:

a) indicação principal é como prótese provisória ${ }^{5}$;

b) para substituir a PPR provisória convencional. É uma alternativa quando não se deseja usar a PPR provisória tradicionalmente confeccionada de acrílico e ganchos de arame $^{5}$;

c) pacientes com dificuldades de abertura da boca e alterações de motricidade, pois este material apresenta facilidade nos movimentos de inserção e remoção devido à sua estrutura flexível ${ }^{6,15}$;

d) pacientes com doença periodontal, que ao terem os tecidos de suporte diminuídos ficam com uma maior quantidade de raiz exposta e neste tipo de prótese as superfícies retentivas não prejudicam nem traumatizam as peças dentárias com forças horizontais ${ }^{6,15}$;

e) pacientes que frequentemente estão expostos a situações violentas como, os atletas, policiais, bombeiros, militares entre outros ${ }^{24}$

f) durante o pós-operatório de implantes, pois a sua característica resiliente diminui a sobrecarga oclusal e melhora a resposta dos tecidos. Contudo, estes fatores devem ser incluídos a uma oclusão balanceada e boa estabilização da prótese provisória ${ }^{5}$;

g) reabilitação de dentes permanentes em pacientes jovens em crescimento ósseo ${ }^{30}$;

h) podem servir como opção aos pacientes que apresentam reações alérgicas aos monômeros das resinas acrílicas convencionais ${ }^{5}$;

i) pacientes com tórus palatino ou mandibular ${ }^{6,30}$.

São contraindicações da PPR Flexível:

a) overbites profundos, de quatro milímetros ou mais, onde os dentes anteriores podem ser deslocados com movimentos extrusivos ${ }^{24}$;

b) pacientes que sofreram excessiva perda óssea, principalmente em zonas anterossuperiores ${ }^{24}$;

c) na reabilitação de arcos de extremos livres, pois, devido à constituição do seu material elástico, estas tendem a efetuar movimentos de báscula ${ }^{30}$;

d) pacientes que apresentem um rebordo muito flácido e com graves problemas de higienização ${ }^{31}$;

e) quando o espaço interoclusal é menor de quatro milímetros na área posterior ${ }^{18}$;

f) devido à ausência de estudos clínicos conclusivos na literatura e algumas controvérsias associadas ao comportamento deste tipo de prótese em longo prazo, não é aconselhável indicá-la como tratamento definitivo ${ }^{5,18}$.

○ VANTAGENS E DESVANTAGENS

VANTAGENS E DESVANTAGENS DAS PPRs CONVENCIONAIS

a) as PPRs provisórias apresentam custo inferior às PPRs flexíveis ${ }^{9}$; 
b) os grampos apresentam maior resistência à fratura desde que sejam bem planejados e executados ${ }^{8}$;

c) promovem melhor distribuição de forças aos dentes pilares devido à presença de apoios e nichos ${ }^{2}$;

d) promovem melhor estabilização dos dentes pilares por apresentarem opositores rígidos ${ }^{32}$;

e) maior condutividade térmica na porção confeccionada com metal ${ }^{24}$;

f) boa estética da porção acrílica por permitir caracterização das bases ${ }^{9}$;

g) maior facilidade de ajuste e polimento ${ }^{9}$;

h) maior facilidade de manutenção da dimensão vertical de oclusão ${ }^{33}$;

i) adesão química aos dentes de acrílico ${ }^{33}$;

j) facilidade de reembasamento e de reparos na porção acrílica $^{24}$.

Desvantagens das PPRs convencionais: aparente

a) estética inferior devido à presença de metal

b) processamento mais complexo, uma vez que necessita da etapa de fundição da armação metálica ${ }^{13}$;

c) base acrílica mais friável ${ }^{10}$;

d) baixa condutividade térmica na porção confeccionada com resina ${ }^{9}$;

e) maior desenho com maior extensão de volume na porção acrílica ${ }^{6}$

f) maior toxicidade, prejudicial aos portadores de reações alérgicas ao monômero ${ }^{5,33}$.

Já as PPRs flexíveis apresentam as seguintes vantagens:

a) estética, que garante que a cor da prótese corresponda à cor da mucosa oral e dispensa o uso da armação metálica ${ }^{13,34}$;

b) satisfação e conforto para o paciente ${ }^{13,18,34}$;

c) por ser translúcida, garante a estética gengival ${ }^{33}$;

d) devido a sua estrutura flexível a fratura dos componentes ocorre com dificuldade, com maior resistência aos inúmeros ciclos de inserção e remoção sem perder a capacidade retentiva e ainda são mais leves que as PPRs convencionais $^{34}$;

e) sua característica resiliente diminui a concentração de forças sobre o rebordo, favorecendo a regeneração e a remodelação óssea pós exodontia e cirurgia de implantes quando comparada a PPR provisória convencional, porém essa afirmação não é verdadeira quando comparada a PPR convencional com apoios ${ }^{32}$;

f) ausência de monômeros, diminuindo a toxicidade e as reações alérgicas ${ }^{35}$;

g) de modo geral, apresentam menor absorção de água do que as resinas acrílicas convencionais e adição de nylon na sua estrutura molecular como ocorrem em algumas marcas comerciais, melhora ainda mais essa característica. Isso confere ao material, característica mais higiênica, menor acúmulo de placa e uma movimentação mais suave dos tecidos bucais ${ }^{18}$. citadas:

Como desvantagens da PPR flexível podem ser

a) possibilidade de danos periodontais ${ }^{34}$;

b) quando são comparados custos, ela é mais onerosa que a PPR provisória convencional e mais barata que a PPR com estrutura metálica ${ }^{34}$;

c) a PPR flexível deve ser usada apenas como caráter estético nos casos pós-cirúrgicos, pois a mastigação de alimentos duros e fibrosos poderá provocar sobrecarga no rebordo alveolar e prejudicar a cicatrização, embora de modo geral sua resposta tecidual seja melhor que a PPR provisória convencional ${ }^{32}$;

d) sua resiliência dificulta a manutenção da dimensão vertical de oclusão adequada ${ }^{33}$;

e) dificuldades de ajustes e polimento ${ }^{33}$;

f) falta de adesão química com os dentes de acrílico, que geralmente refletem no descolamento dessas estruturas ${ }^{32}$

g) alteração de cor ao longo do tempo ${ }^{12,18}$;

h) baixa condutibilidade térmica ${ }^{24}$;

i) em pessoas com grande capacidade muscular e mordida muito forte os dentes artificias estão sujeitos a pequenas deslocações durante a mastigação pela ligeira flexibilidade do material no nível dos colos dentários, produzindo sons desagradáveis para o paciente e à sua volta $^{30}$ $\operatorname{reparos}^{24,30}$

j) apresentam dificuldade para reembasamentos e

\section{CONCLUSÃO}

As PPRs flexíveis são constituídas por material flexível e dentes artificiais. Sua principal vantagem está no fato de não apresentarem metal em sua composição, sendo, portanto, mais estéticas. Porém, elas não substituem as PPRs convencionais, principalmente em arcadas de extremidade livre, em que a estabilidade depende de uma combinação de dentes e tecidos mole. Desta forma, as próteses flexíveis devem ser utilizadas de forma provisória e de acordo com as situações mencionadas anteriormente. As realizações da manutenção e de consultas regulares devem ser feitas de qualquer maneira, pois ainda existem poucos relatos na literatura sobre seu uso em longo prazo, por isso são necessários mais estudos e pesquisas cientificas a respeito das PPRs flexíveis.

\section{REFERÊNCIAS}

1. Hamanaka I, Iwamoto M, Lassila L, Vallittu P, Shimizu $\mathrm{H}$, Takahashi Y. Influence of water sorption on mechanical properties of injection-molded thermoplastic denture base resins. Acta Odontol Scand. 2014; 72(8):859-65.

2. Carr AB, Brown D, Cooper SEM. Prótese parcial removível. 12. ed. São Paulo: Elsevier; 2012.

3. Modaffore P. O que devemos enviar ao laboratório para confecção de uma boa prótese parcial removível. J Bras Clín Estet Odontol. 2010; 4(24):77-81.

4. Shaghaghian S, Taghva M, Abduo J, Bagheri R. Oral health-related quality of life of removable partial denture wearers and related factors. J Oral Rehabil. 2014; 42(1):40-8.

5. Chikunov I, Doan P, Vahidi F. Implant-retained partial overdenture with resilient attachments. J Prosthodont 2008; 17(2):141-8.

6. Da Costa RFA. Próteses flexíveis em poliamida: revisão da literatura [dissertação]. Porto: Faculdade de Medicina Dentária, Universidade do Porto; 2016.

7. Fueki K, Ohkubo C, Yatabe M, Arakawa I, Arita M, Ino S. Clinical application of removable partial dentures using thermoplastic resin - Part I: Definition and indication of non-metal clasp dentures. J Prosthodont Res. 2014; 58(1):3-10.

8. Goiato MC, Santos DM, Haddad MF, Pesqueira AA. Effect of accelerated aging on the microhardness and color stability of flexible resins for dentures. Braz Oral 
Res. 2010;24(1):114-119.

9. Fueki K, Ohkubo C, Yatabe M, Arakawa I, Arita M, Ino $S$ et al. Clinical application of removable partial dentures using thermoplastic resin - Part II: Material properties and clinical features of non-metal clasp dentures. J Prosthodont Res. 2014; 58(2):71-84.

10. Abhay PN, Karishma S. Comparative evaluation of impact and flexural strength of four commercially available flexible denture base materials: An in vitro study. J Indian Prosthodont Soc. 2013;13(4):499-508.

11. Goiato MC, Panzarini SR, Tomiko C, Luvizuto ER Temporary flexible immediately removable partial denture: a case report. Dent Today. 2008; 27(3):114-6.

12. Wada J. A comparison of the fitting accuracy of thermoplastic denture base resins used in non-metal clasp dentures to a conventional heat-cured acrylic resin. Acta Odontol Scand. 2015; 73(1):33-7.

13. Paulino JS. PPR Flexível - características, vantagens e desvantagens em relação à PPR convencional [trabalho de conclusão do curso]. São José do Rio Preto: ETEC Philadelpho Gouvêa Netto; 2009.

14. Nishimori L, Tomazini TF, Progiante PS, Marson FC, Silva CO, Corrêa GO, et al. Estética das Prótese Flexíveis: Relato De Caso Clinico. Braz J Surg Clin Res. 2013; 5(3):37-40

15. Carr AB, Mcgivney GP, Brown D T. McCracken's Removable partial prosthodontics. 11. ed. St Louis: Elsevier Mosby; 2005.

16. Kaplan P. Flexible removable partial dentures: design and clasp concepts. Dent Today. 2008; 27(12):120-3.

17. João M, Lacroix SP. Dicas de Materiais. J ABORJ. 2005:1.

18. Singh K, Aeran H, Kumar N, Gupta N. Flexible thermoplastic denture base materials for aesthetical removable partial denture framework. J Clin Diagn Res. 2013: 7(10):2372-3.

19. Gift HC, Drury TF, Nowjack-Raymer RE, Selwitz RH. The State of the Nation's Oral Health: Mid-decade Assessment of Healthy People 2000. J Public Health Den. 1996; 56(2):84-91.

20. Thakral GK. Flexible partial dentures- A hope for challenged mouth. People's. J Scientific Res. 2012; 5(2): 55-59.

21. Stegun R. Resolução da perda dentária anterior com prótese flexível. 2009. Disponível em: <www.envelhecersorrindo.com.br>. Acesso em: 20 ago. 2014.

22. Meijer GJ, Wolgen PJ. Provisional flexible denture to assist in undisturbed healing of the reconstructed maxila. J Prost Dent. 2007; 98(4):327-8.

23. Noort R. Introdução aos Materiais Dentário. 3. ed. Rio de Janeiro: Mosby Elsevier; 2007. p.127-130.

24. Takabayashi Y. Characteristics of denture thermoplastic resins for non-metal clasp dentures. Dent Mat J. 2010; 29(4):353-61.

25. De Paula V, Lorenzoni FC, Bonfante G. Próteses Parciais Removíveis não Convencionais - Parte I. Full Dent Sci. 2011; 2(7):284-93.

26. Giampaolo ET, Leonardi P, Cucci ALM, Fuller JB. Grampos de retenção para prótese parcial removível. Análise da resistência à flexão em função de grampos, ligas e técnicas de fusão. Rev Odontol UNESP; 1991; 20:293-298.

27. Hazari P, Bhoyar A, Mishra SK, Yadav NS, Mahajan H. A Comparison of Masticatory Performance and Efficiency of Complete Dentures Made with High
Impact and Flexible Resins: A Pilot Study. J Clin Diagn Res. 2015; 9(6):ZC29-ZC34.

28. Murthy V, Yuvraj V, Nair PP, Thomas S. Prosthodontic management of radiation induced xerostomic patient using flexible dentures. BMJ Case Reports 2012; doi: 10.1136/bcr.11.2011.5250

29. Phoenix RD, Cagna DR, Defreest CF. Prótese Parcial Removível: Clínica de Atewart. 3 ed. São Paulo: Quintessence; 2007.

30. Hemmati MA, Vafaee F, Allahbakhshi H. Water sorption and flexural strength of thermoplastic and conventional heat-polymerized acrylic resins. J Dent. 2015; 12(7):478-84.

31. Hamanaka I, Iwamoto M, Lassila LV, Vallittu PK, Shimizu H, Takahashi Y. The effect of cycling deflection on the injection-molded thermoplastic denture base resins. Acta Odontol Scand. 2016; 74(1):67-72.

32. Sharma A, Shashidhara HS. A review: Flexible removable partial dentures. J Dent Med Sci. 2014; 13(12):58-62.

33. Hiskin S. Prótesis Flexibles de Nylon Eemovibles. Rev Nac Odontol México. 2010; 4(2):7.

34. Negrutiu M. Thermoplastic resins for flexible framework Removable Partial Dentures. Timisoara Med J. 2005;55(3):295-99.

35. Hamanaka I, Takahashi Y, Shimizu H. Mechanical properties of injection-molded thermoplastic denture base resins. Acta Odontol Scand. 2004; 69(2):75-9.

\section{CONFLITO DE INTERESSES}

Os autores declaram não haver conflitos de interesse.

\section{AUTOR PARA CORRESPONDÊNCIA}

\section{Marcela Filié Haddad}

marcela.haddad@unifal-mg.edu.br

Submetido em 07/03/2017 Aceito em 14/06/2017 